\title{
Angioarchitecture of Transverse-Sigmoid Sinus Dural Arteriovenous Fistulas: Evaluation of Shunted Pouches by Multiplanar Reformatted Images of Rotational Angiography
}

\author{
H. Kiyosue, S. Tanoue, M. Okahara, Y. Hori, J. Kashiwagi, Y. Sagara, T. Kubo, and H. Mori
}

\begin{abstract}
BACKGROUND AND PURPOSE: Recognition of shunted pouches dural arteriovenous fistula allows us to treat the disease effectively by selective embolization of the pouches at first. However, the shunted pouches in transverse-sigmoid sinus dural arteriovenous fistulas have not been well-documented. Our aim was to evaluate the angioarchitecture of transverse-sigmoid sinus dural arteriovenous fistulas, including the frequency and location of shunted pouches and their feeding arteries.
\end{abstract}

MATERIALS AND METHODS Twenty-five consecutive cases of TSS-DAVFs that underwent rotational angiography and transvenous embolization between 2008 and 2011 were reviewed. Multiplanar reformatted images of rotational angiography and selective angiography were reviewed with a particular focus on the shunted pouches.

RESULTS: All 25 cases showed SPs, with numbers ranging from 1 to 4 pouches (mean, 2.35). The SPs were located at the transverse-sigmoid junction in 16, close to the vein of Labbé in 9, at the dorsal-to-sigmoid sinus in 9, inferior to the sigmoid sinus in 6, at the sigmoid-jugular junction in 5 , and inferior to the transverse sinus or the sinus confluence in 14 . The SP at the sigmoid sinus was frequently fed by the jugular branch of the ascending pharyngeal artery and the stylomastoid artery. The SP at the transverse-sigmoid junction and the vein of Labbé was fed by the petrosal/petrosquamous and posterior branches of the middle meningeal artery and the transosseous branches of the occipital artery. The SP inferior to the transverse sinus and the sinus confluence was fed by the transosseous branches of the occipital artery and the posterior meningeal artery. All cases were successfully treated by transvenous embolization with sinus packing $(n=13)$ or selective embolization of the SP $(n=12)$.

CONCLUSIONS: The presence of SP is a common angioarchitecture of TSS-DAVFs. Identification of the SPs would be useful for their treatment.

ABBREVIATIONS: $\mathrm{AVF}=$ arteriovenous fistula; $\mathrm{MPR}=$ multiplanar reformatted; $n-\mathrm{BCA}=$ butyl 2 -cyanoacrylate; $\mathrm{SP}=$ shunted pouch; TSS-DAVF $=$ transversesigmoid sinus dural arteriovenous fistula

T ransverse-sigmoid sinus dural arteriovenous fistula is one of the most common types of intracranial DAVFs and can lead to various disorders, from tinnitus to fatal cerebral hemorrhage. A majority of TSS-DAVFs have been treated by endovascular techniques, including transarterial embolization and/or transvenous embolization. The drainage pattern of the TSS-DAVFs is strongly related to the clinical symptoms; therefore, the importance of evaluating the drainage routes of the TSS-DAVF before treatment has been well-recognized. ${ }^{1,2}$ However, other

Received March 11, 2012; accepted after revision September 28

From the Departments of Radiology (H.K., S.T., M.O., J.K., Y.S., H.M.) and Neurosurgery (T.K.), Oita University Faculty of Medicine, Yufu City, Oita Japan; and Department of Radiology (Y.H.), Nagatomi Neurosurgical Hospital, Oita, Japan.

Please address correspondence to Hiro Kiyosue, Department of Radiology, Oita University, Idaigaoka, Hasama, Yufu City, Oita, Japan 879-5963; e-mail: hkiyosue@ oita-u.ac.jp

http://dx.doi.org/10.3174/ajnr.A3428 angioarchitectures, such as fistulous points and dural sinus compartments, have not been well-documented. Some cases of TSS-DAVFs can be successfully treated by selective embolization of a fistulous portion with preservation of the normal sinus lumen. ${ }^{3-5}$ Although these cases are generally thought to be special, 1 study showed that the fistulous compartment of the dural sinus often exists in and around the dural sinus in TSS-DAVFs. ${ }^{6}$

Recent developments in 3D angiography technology allow us to evaluate the angioarchitecture of DAVFs more easily and precisely. ${ }^{7}$ In this study, we evaluated the angioarchitecture of TSSDAVFs, including the frequency and location of these fistulous compartments of dural sinuses on the basis of MPR images of rotational angiography. Additionally, we discuss the endovascular techniques for the treatment of TSS-DAVFs. Several different terms have been used to refer to these fistulous portions located outside the main lumen of the dural sinus, including venous re- 
Table 1: Characteristics of the 25 patients

\begin{tabular}{lr}
\hline \multicolumn{1}{c}{ Characteristics } & \\
\hline Sex & 17 \\
Male & 8 \\
Female & 12 \\
Symptoms & 13 \\
Tinnitus & 6 \\
Consciousness disturbance & 4 \\
Headache & 2 \\
Cerebral hemorrhage & 2 \\
Visual deficits & 1 \\
Hemiparesis & 2 \\
Dizziness & \\
No symptom & 5 \\
Types of DAVFs & 5 \\
Type I & 9 \\
Type lla & 6 \\
Type lla+b & \\
Type llb & 12 \\
Transvenous embolization & 13 \\
Selective embolization & \\
Sinus packing &
\end{tabular}

cipients of DAVFs, dural sinus septations, accessory sinuses, parallel channels, parallel sinuses, and fistulous drainage. In this article, we use the term "shunted pouch" to refer to these structures.

\section{MATERIALS AND METHODS}

We retrospectively analyzed 25 consecutive patients with TSSDAVFs who underwent rotational cerebral angiography between June 2008 and December 2011. We reviewed their imaging findings and interventional procedures. Characteristics of the 25 patients are summarized in Table 1. The patient ages ranged from 47 to 84 years (mean age, 66 years), and there were 17 men and 8 women. The TSS-DAVFs were classified into the following 4 types according to the revised classification of Djindjian and Merland, depending on the type of venous drainage ${ }^{2}$ : type I, antegrade drainage into the sinus; type IIa, retrograde drainage into the sinus; type IIa $+\mathrm{b}$, retrograde drainage into the sinus and cortical veins; and type IIb, retrograde cortical venous drainage. There were 5 type I AVFs, 5 type IIa AVFs, 9 type IIa +b AVFs, and 6 type IIb AVFs. All 25 patients were treated by transvenous embolization alone or combined with transarterial embolization with glue. Selective digital subtraction angiography of the bilateral internal and external carotid arteries and the vertebral arteries was performed in all patients by using biplane angiography equipment (Innova3131; GE Healthcare, Milwaukee, Wisconsin; Infinix VB, Toshiba Medical, Tokyo, Japan). Each patient gave written informed consent before the examination.

Anteroposterior, lateral, and oblique views of selective biplane cerebral angiography of the bilateral external carotid arteries and their main feeding branches, the internal carotid arteries, and the vertebral artery were obtained by using a $4 \mathrm{~F}$ diagnostic catheter inserted via the right femoral artery. When AVFs were found by using biplane selective angiography of each cerebral artery, rotational angiography was subsequently performed. The rotational angle was $200^{\circ}$, and the rotational speed of the C-arm was $40^{\circ}$ / (Innova3131) or 50\% (Infinix VB). Data were acquired in a $512 \times$ 512 matrix by using a $20-\mathrm{cm}$ FOV flat panel detector. Nonionic

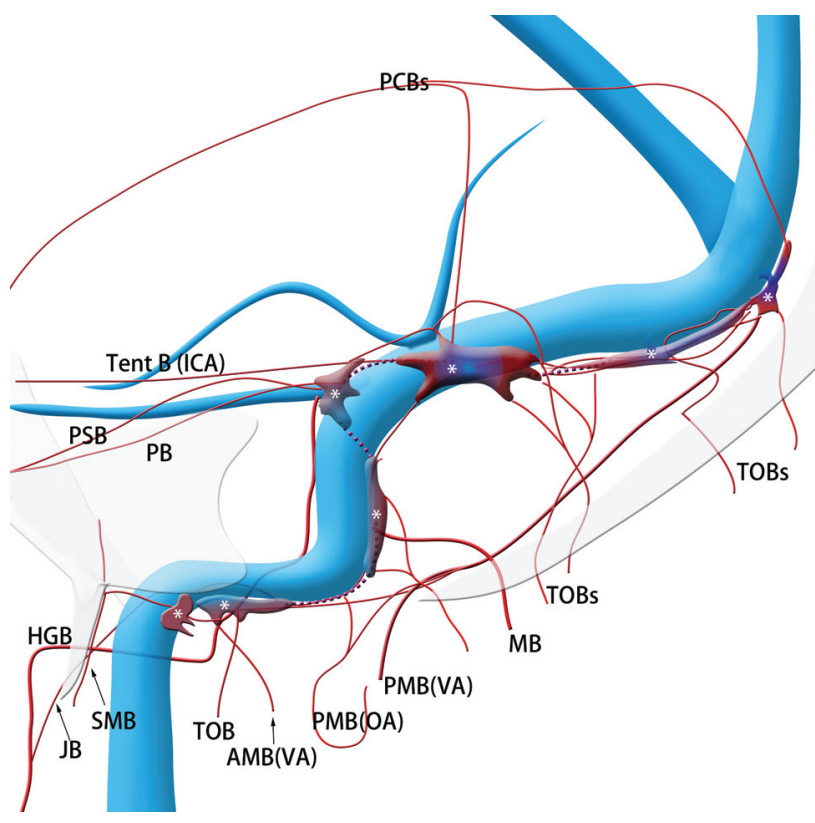

FIG 1. Schematic of the shunted pouches and their feeding arteries in transverse-sigmoid sinus dural arteriovenous fistulas. Purple indicates shunted venous pouches; APA, ascending pharyngeal artery; OA, OCcipital artery, MMA, middle meningeal artery; PAA, posterior auricular artery; VA, vertebral artery; SMB, stylomastoid branch of the $O A ; M B$, mastoid branch of the OA; TOB, transosseous branches of the OA; JB, jugular branch of the APA; PB, petrosal branch of the MMA; PSB, petrosquamous branch of the MMA; Post $B$, posterior convexity branch of the MMA; PMA, posterior meningeal artery from the VA: Tent B, tentorial branch from the ICA; SPS, superior petrosal sinus; LTS, lateral tentorial sinus.

Table 2: Locations and frequency of the shunted venous pouch in 25 patients with transverse sigmoid sinus dural arteriovenous fistulas

\begin{tabular}{lr}
\hline \multicolumn{1}{c}{ Locations } & No. of Cases (\%) \\
\hline Sigmoid-jugular junction & $5(20 \%)$ \\
Horizontal portion of SS & $6(24 \%)$ \\
Perpendicular portion of SS & $9(35 \%)$ \\
Trans-sigmoid junction & $16(64 \%)$ \\
Junction of vein of Labbé & $9(36 \%)$ \\
Distal portion of TS & $8(32 \%)$ \\
Sinus confluence & $6(24 \%)$ \\
\hline
\end{tabular}

Note:- - S indicates sigmoid sinus; TS, transverse sinus.

iodinated contrast material (iopamidol, Iopamiron 300; Bayer Healthcare Japan, Osaka, Japan) was injected intra-arterially at a flow rate of $1.5-3.5 \mathrm{~mL} / \mathrm{s}(14-24.5 \mathrm{~mL}$ of total volume) through an automatic injector, and the injection was initiated 1.5-2.0 seconds before the rotation. 3D images with maximum intensity projection and volume-rendering reconstruction and MPR images composed of sections with 0.3 - to $1-\mathrm{mm}$ thickness and a $0.5-\mathrm{mm}$ interval were obtained from the data of rotational angiography by using a workstation (Advantage Workstation; GE Healthcare and Ziostation; Ziosoft, Tokyo, Japan).

All angiographic and MPR images were reviewed by 2 experienced neuroradiologists (H.K. and S.T.) to reach a consensus regarding the presence and locations of SPs as well as the feeding arteries for each SP. MR angiography, postcontrast fat-suppressed 3D gradient-echo MR imaging, and selective venography during transvenous embolization were also reviewed when available. A 

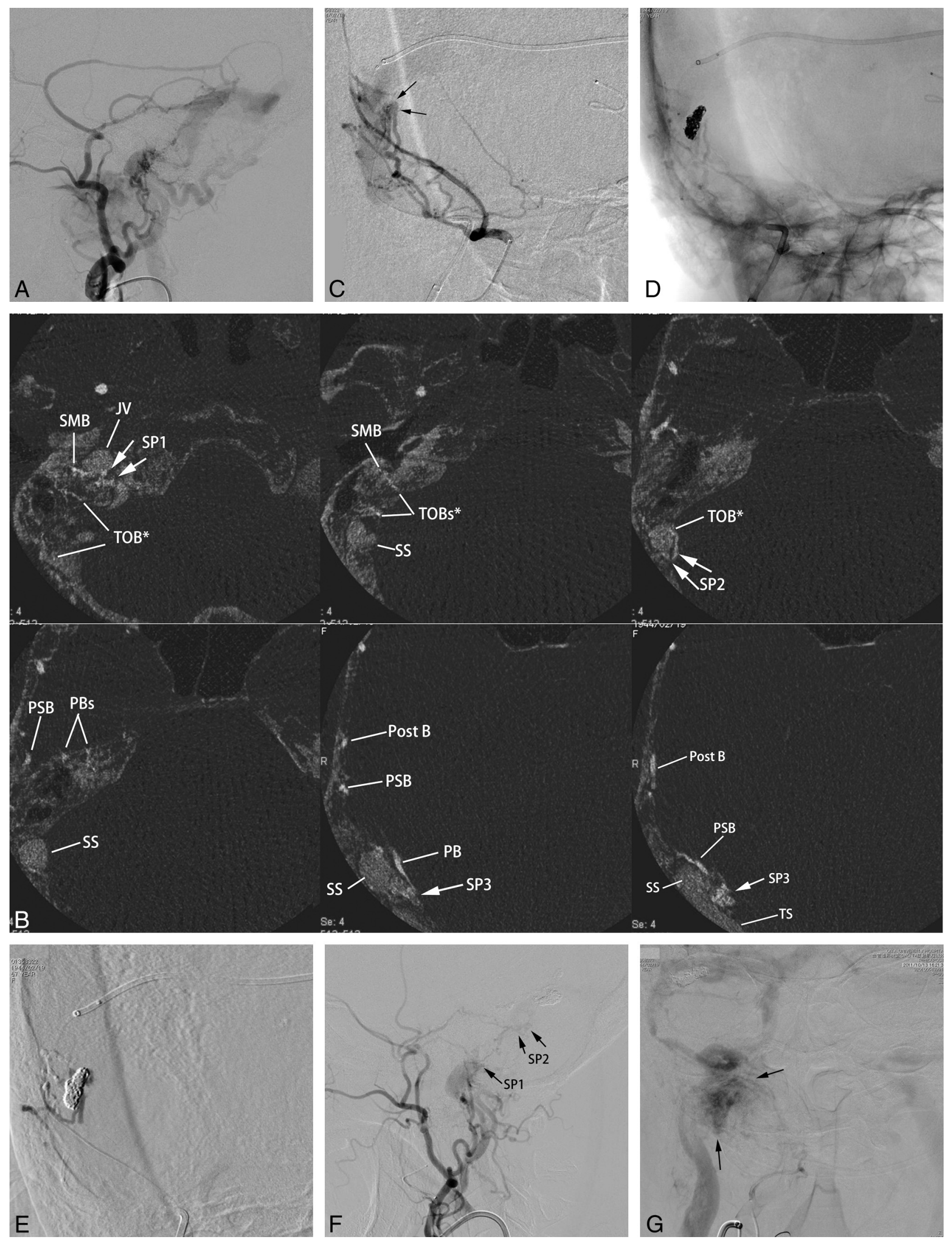

FIG 2. See legend on next page. 
shunted pouch was defined as a tubular or elliptic vascular structure that is separated from the main sinus lumen into which multiple feeding arteries converge and continue to the TSS.

\section{The Findings of a Shunted Pouch on MPR Images Included the Following:}

1) Convergence of the feeding arteries. We tracked each feeding artery from its origin by paging through the MPR images on a viewer and observing multiple feeders converging to a vascular pouch.

2) Separation from the main lumen of the dural sinus. The shunted pouch is clearly separated from the main lumen of the dural sinus by a low-attenuation linear structure (septation), and/or it protruded from the sinus.

3) Caliber change at the fistulous point. The shunted pouch is larger than all of its feeding arteries.

4) Contrast gradient and continuity to the main lumen of the dural sinus. Contrast media injected from the feeding artery fills the shunted pouch, and the contrast media are diluted by blood from other feeders. The shunted pouch is connected to the main lumen of the dural sinus via a single channel or multiple channels, and the contrast gradient is observed in and around the outlet area.

Locations and shapes of a shunted pouch and its relationship with feeding arteries on MPR images were correlated with those findings of selective angiography and/or selective venography during transvenous embolization.

Transvenous embolization with sinus packing $(n=13)$ or selective embolization $(n=12)$ was performed in all cases. Selection of the techniques mainly depended on the types of DAVFs and the accessibility of the microcatheter into the shunted pouches. Even in cases treated by sinus packing, selective occlusion of the shunted pouch was performed first to reduce the shunted blood flow. Follow-up DSA was performed at 3-6 months after embolization, and MR angiography was performed at 3 months and every 6 months after embolization.

Institutional review board approval is not required for retrospective studies at our institution.

\section{RESULTS}

Shunted pouches were found in all 25 patients, and the number of the shunted pouches in each case ranged from 1 to 4 (mean, 2.35).
The mean number of shunted pouches in each type of DAVF was 1.8 for type I; 2.8 for type IIa; 2.56 for type II $+\mathrm{b}$; and 2.16 for type IIb. Type IIa and type IIa $+\mathrm{b}$ had a larger number of shunted pouches. However, these types did not have a statistically significantly higher number than the other types of DAVFs.

The locations of the shunted pouches and their feeding arteries are summarized in Fig 1 and Table 2. The shunted pouches were located superomedial to the transverse sigmoid junction in 16 cases, close to the vein of Labbé in 9 cases, dorsal to the vertical portion of the sigmoid sinus in 9 cases, inferior to the horizontal portion of the sigmoid sinus in 6 cases, around the sigmoid-jugular junction in 5 cases, inferior to the distal portion of the transverse in 8 cases, and inferior to the sinus confluence in 6 cases (Figs 2-6). There is a close relationship between the location of the shunted pouches and feeding arteries. The shunted pouches around the sigmoid sinus and the sigmoid-jugular junction were frequently fed by the jugular branch of the ascending pharyngeal artery ( 11 of 20 cases) and the stylomastoid artery ( 7 of 20 cases) (Figs 1-3, 6). Both of the shunted pouches at the transverse-sigmoid junction and the vein of Labbé were fed by transosseous branches of the occipital artery (20 of 25 cases) and the middle meningeal artery. The former was more often supplied by the petrosal branch (12 of 16 cases) and/or the petrosquamous branches ( 12 of 16 cases) of the middle meningeal artery than the latter ( 3 of 9 cases and 4 of 9 cases) (Figs 4 and 5). The shunted pouches inferior to the transverse sinus or the sinus confluence were fed by the transosseous branches of the occipital artery ( 12 of 13 cases), the posterior branch of the middle meningeal artery ( 5 of 13 cases), and the posterior meningeal artery (8 of 13 cases) (Figs 3 and 4).

All cases were successfully treated by transvenous embolization with sinus packing $(n=13)$ or selective embolization of the shunted pouch $(n=12)$. In 10 cases of DAVFs without cortical reflux (type I and type IIa), 9 cases were treated by selective transvenous embolization. One case of type I DAVF, in which the affected sinus did not receive cerebral venous drainage, was treated by sinus packing. In 9 cases of type IIa $+\mathrm{b}$ DAVFs, 3 were treated by selective embolization and 6 were treated by sinus packing. All 6 cases of type IIb DAVFs were treated by sinus packing. Among the 12 cases treated by selective transvenous embolization, 6 patients undergoing transarterial embolization with $n$-butyl 2-cyanoacrylate underwent concurrent selec-

FIG 2. Type lla transverse-sigmoid sinus dural arteriovenous fistulas with multiple shunted pouches. The DAVFs were associated with paraganglioma at the ipsilateral carotid bifurcation. A, Lateral view of right external carotid angiography shows DAVFs involving the transverse and sigmoid sinuses. The DAVFs are fed by the middle meningeal artery, the posterior auricular artery, and the occipital artery and drain into the right jugular vein and the contralateral transverse sinus. B. The axial reformatted images show multiple SPs (SP 1-3), which are located at the sigmoid-jugular junction (SP1), mediodorsal to the vertical portion of the sigmoid sinus (SP2), and at the transverse-sigmoid junction (SP3), respectively. The transosseous feeders from the stylomastoid branches (SMB) of the posterior auricular arteries supply the SP1; the transosseous branch (TOB) of the occipital artery and the posterior auricular artery supply the SP2. The petrosal branch (PB), the petrosquamous branch (PSB), and the posterior convexity branch (Post B) of the middle meningeal artery supply the SP3. C, Anterior oblique view of selective angiography of the middle meningeal artery during the embolization procedure shows a shunted pouch (arrows) that is located medial to the transversesigmoid junction. Multiple branches of the middle meningeal artery converge on the shunted pouch. A microcatheter is advanced into the shunted pouch with a transvenous approach. $D$. Fluoroscopic image after selective embolization shows a coil mass in the shunted pouch. Minimal shunt through the coil mass remains after selective transvenous embolization. $E$, Angiography during injection of diluted $n$-butyl 2 -cyanoacrylate ( $33 \% n$-BCA-lipiodol mixture) via the petrosquamous branch shows sufficient filling of the $n$-BCA into the residual part of the shunted pouch without migration into the main lumen of the sinus. F, Lateral view of right external carotid angiography after embolization shows marked regression of DAVFs with their disappearance at the transverse-sigmoid junction. Small residual AVFs were seen at the vertical portion of the sigmoid sinus (SP2) and the sigmoid-jugular junction (SPI). These residual shunted pouches were not treated because they seemed to be too small to catheterize and were supplied by dangerous feeders of stylomastoid branch and a petrosal branch of the middle meningeal artery. G, Frontal view of common carotid angiography at the venous phase shows that normal blood flow via the right transverse-sigmoid sinus is preserved. Arrows indicate staining of the paraganglioma at the carotid bifurcation. 

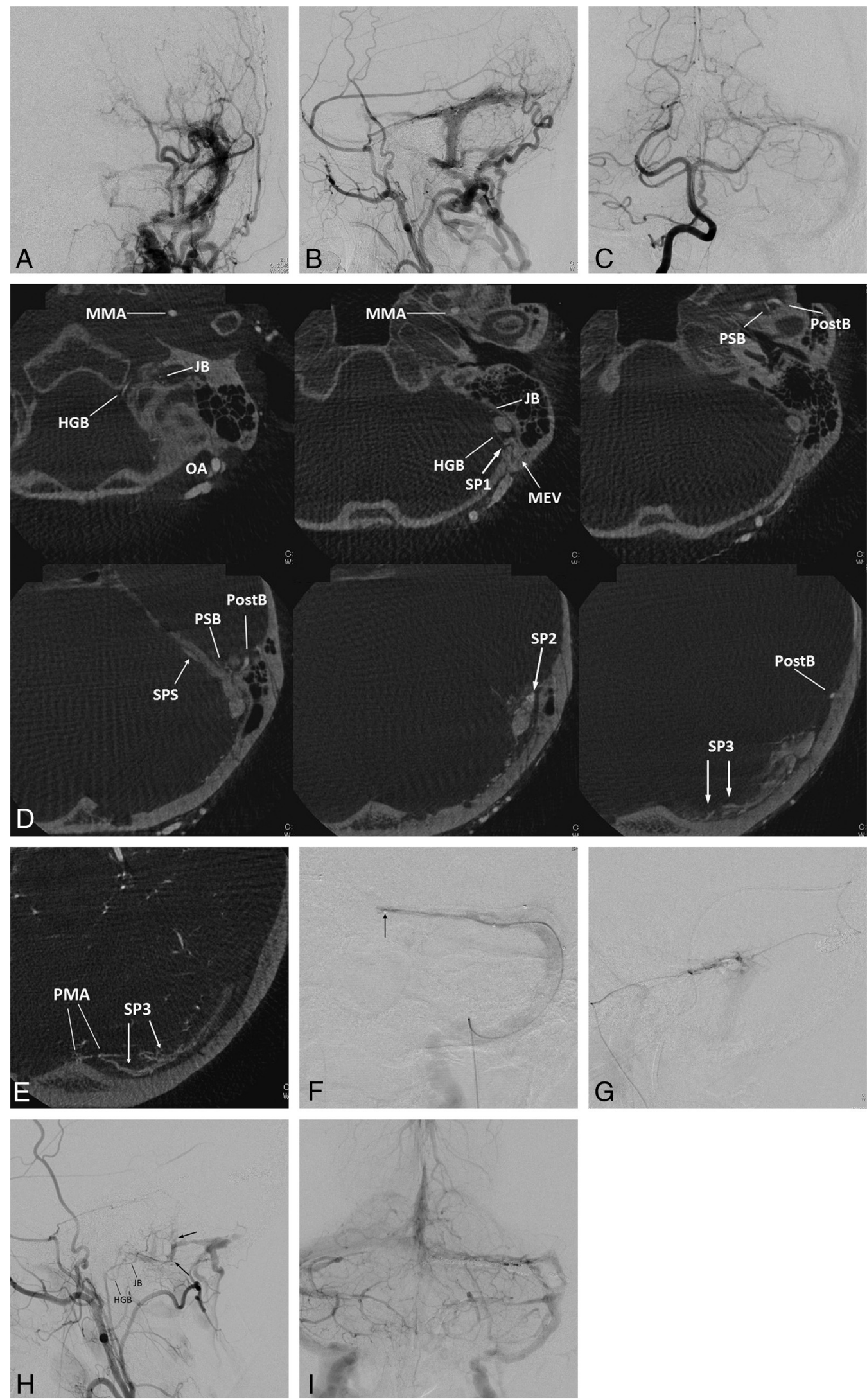

FIG 3. See legend on next page. 
tive transvenous embolization, and balloon angioplasty of the occluded sinuses was performed in 3 patients.

All cases were successfully treated without any complications. DAVFs disappeared in all 13 cases treated by sinus packing. In 12 cases treated by selective transvenous embolization, the DAVFs disappeared in 6 cases and markedly regressed in 6 cases, and the main lumen of the dural sinuses was successfully preserved or reconstructed in all 12 cases. Except for 2 asymptomatic cases, symptoms disappeared or markedly improved in 22 patients. The symptoms were not significantly improved in 1 patient who had a massive venous infarction before treatment. During 29 months of mean follow-up (range, 4-50 months), 23 patients showed stable occlusion (no recurrence, further regression, or stable remnant) of the DAVFs. In 1 patient, the DAVFs developed at another location (midportion of the superior sagittal sinus) at 10 months after transvenous embolization. However, the treated TSS-DAVFs remained occluded. The newly developed DAVFs disappeared by transarterial embolization with $n$-BCA. In another patient with minimal residual DAVFs immediately after selective transvenous embolization, the recurrence of tinnitus symptoms with re-aggravation of the TSSDAVFs was observed at 6 months, and the patient was successfully re-treated by selective transvenous embolization with preservation of the patency of the dural sinus.

\section{DISCUSSION}

Mironov $^{3}$ first described 2 cases of selective transvenous embolization of DAVFs, including 1 superior sagittal sinus DAVF and 1 TSSDAVF. Since then, several cases of intracranial DAVF shunting to a localized venous pouch adjacent to the major dural sinuses have been reported, which can be treated by selective transvenous embolization while preserving the sinus drainage from normal cerebral veins. These are generally thought to be special cases of intracranial DAVF. ${ }^{4}$ Of 140 cases of TSS-DAVFs, Caragine et $\mathrm{al}^{5}$ described 10 patients with TSS-DAVFs with a fistulous venous channel in a position parallel to the TSS (parallel venous channel). However, we often observed similar parasinusal venous pouches in many cases of intracranial DAVFs in our clinical practice. Piske et $\mathrm{al}^{6}$ investigated cerebral angiography in 40 intracranial DAVFs and found a dural sinus compartment in 12 of the 40 cases (30\%). Nine of these cases could be treated by a selective transvenous embolization technique, preserving the patency of the dural sinuses.

In our study, all 25 cases of TSS-DAVFs showed at least 1 shunted pouch on MPR images of rotational angiography. This result suggests that the presence of shunted pouches is a common feature of TSS-DAVF angioarchitecture. The higher incidence of locating shunted pouches was due to an analysis method by using high-resolution cross-sectional images, which clearly demonstrated the angioarchitecture of shunted pouches where multiple feeding arteries converge without overlapping vessels. Hiu et $\mathrm{al}^{7}$ demonstrated the superiority of CT-like images to 2D-DSA in assessing several angioarchitectures of DAVFs, such as feeding arteries and shunting points. In our study, we modified our routine 3D-DSA techniques to demonstrate the angioarchitecture of the DAVFs in detail. We used the following techniques: a selected FOV, thin-section reconstruction with a high-frequency enhancement algorithm, an adequate time delay from contrast injection to rotation, and prolonged injection time. These technical modifications contributed to a good visualization of the angioarchitecture of DAVFs.

Piske et $\mathrm{al}^{6}$ demonstrated 2 types of shunted pouches. One is a restricted part of the dural sinus called "septation," and the other type is a venous channel located outside the sinus called the "accessory sinus." We found several cases of an "accessory sinus," which were located distant from the dural sinus, and we also found some cases of "septation," in which MPR images clearly depicted a septum in the dural sinus. However, we could not distinguish these 2 types in many cases when the shunted pouch was located adjacent to the main lumen of the sinus because the size of the TSS varied individually, and we could not observe the sinus wall by using imaging. In the embryologic development of the dural sinus, the primitive dural venous plexus is formed by dural veins, some of which receive blood from dural osseous components, while others are contiguous with pial veins. Among these dural venous plexuses, the plexus at the base of the dural folds fuse to form the major dural venous sinus. ${ }^{8}$ Therefore, incomplete fusion of these dural plexuses can form compartment "septations" inside the sinus, and the remnant of the dural plexus around the sinus can become an "accessory sinus."

Selective transvenous embolization of the shunted pouch is one of the useful techniques for the treatment of DAVFs without cortical venous reflux (type I and IIa), which can occlude the DAVF while preserving the sinus drainage. Even for the treatment of DAVFs with cortical venous reflux (type IIb and IIa + b) by transvenous sinus packing, selective occlusion of the shunted pouch can initially reduce the shunting blood flow before sinus

FIG 3. Left transverse sigmoid DAVFs (type Ila) with 3 shunted venous pouches. Frontal $(A)$ and lateral $(B)$ views of the left external carotid angiography show DAVFs involving the left transverse-sigmoid sinuses. The DAVFs are fed by multiple branches of the external carotid artery and drain into the posterior condylar vein, the mastoid emissary vein, and the superior petrosal sinus with occlusion of the sigmoid sinus at the proximal end. Frontal view of right vertebral angiography $(C)$ shows the DAVF fed by the posterior meningeal artery. D, Axial reformatted images of rotational angiography of the left external carotid artery show 3 shunted pouches, which are located dorsal to the vertical portion of the sigmoid sinus (SP1), at the transverse sigmoid junction (SP2), and inferior to the distal transverse sinus (SP3). The SP1, SP2, and SP3 are fed by the jugular branch (JB) and hypoglossal branch (HGB) of the ascending pharyngeal artery, the petrosquamous branch (PSB) of the middle meningeal artery, and the posterior convexity branches of the middle meningeal artery and the transosseous branches of the occipital artery, respectively. $E$, An axial reformatted image of rotational angiography of the right vertebral artery shows the posterior meningeal artery (PMA) also supplying the shunted pouch inferior to the distal portion of the transverse sinus (SP3). F, Selective venography of the shunted pouch (SP3) via a microcatheter (arrow) selectively introduced into the SP3 shows the separated venous pouch parallel to the transverse sinus. The shunted pouch was embolized with coils. $G$, Selective angiography with simultaneous injection via 2 microcatheters, one positioned at the petrosquamous branch of the middle meningeal artery and the other positioned at another shunted pouch of SP2, shows a shunted venous pouch at the transverse-sigmoid junction. The shunted pouch was also embolized with coils and glue. $H$, Lateral view of the right external carotid angiography after embolization shows marked regression of the DAVFs. A small part of the DAVFs remains with a shunted venous pouch (arrows) located dorsal to the sigmoid sinus (SPI), which was supplied by a hypoglossal branch (HGB) and a JB of the ascending pharyngeal artery. I, Right vertebral angiography during the venous phase shows coils placed parallel to the transverse sinus. Normal sinus flow of the transverse-sigmoid sinuses is preserved. 

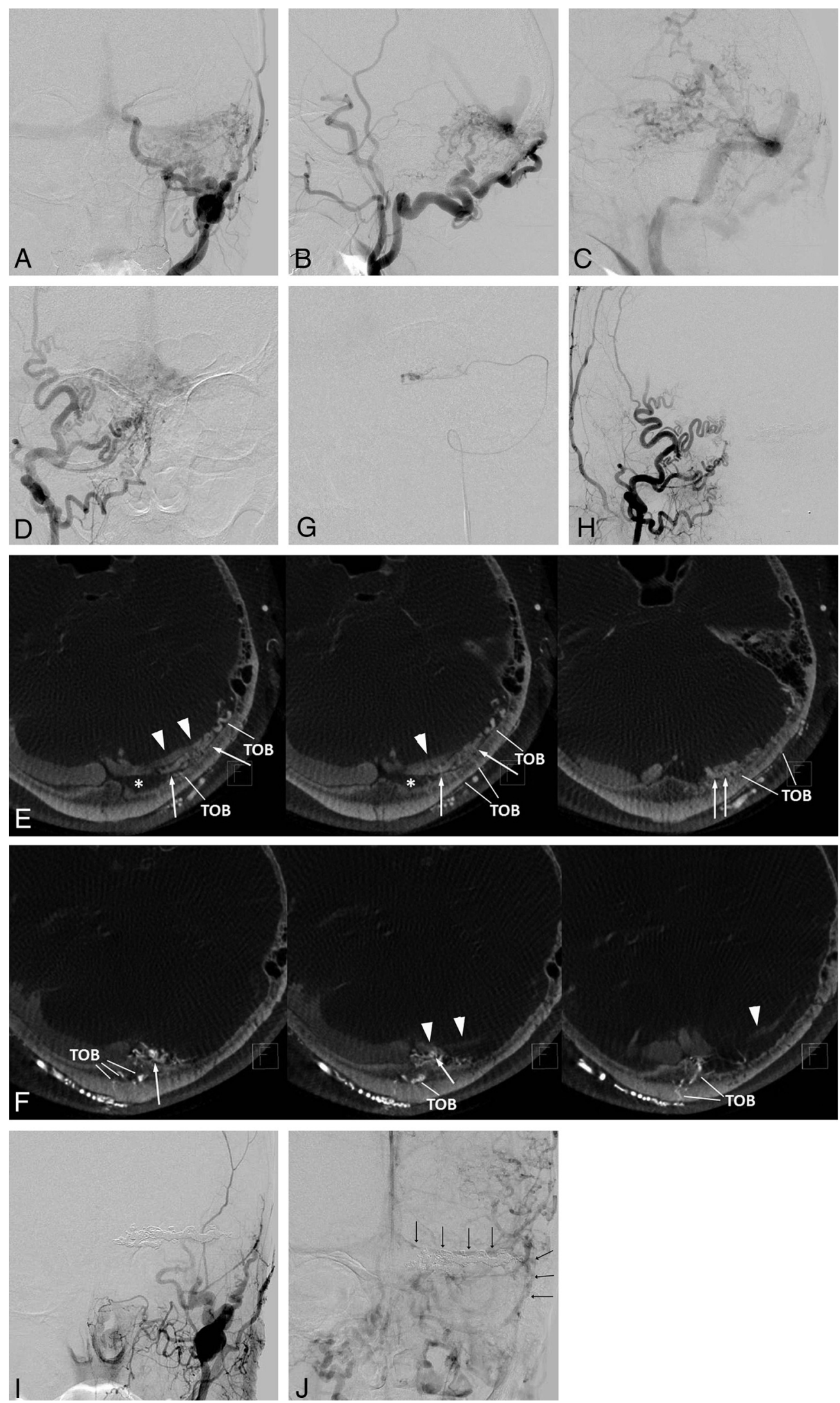

FIG 4. See legend on next page. 

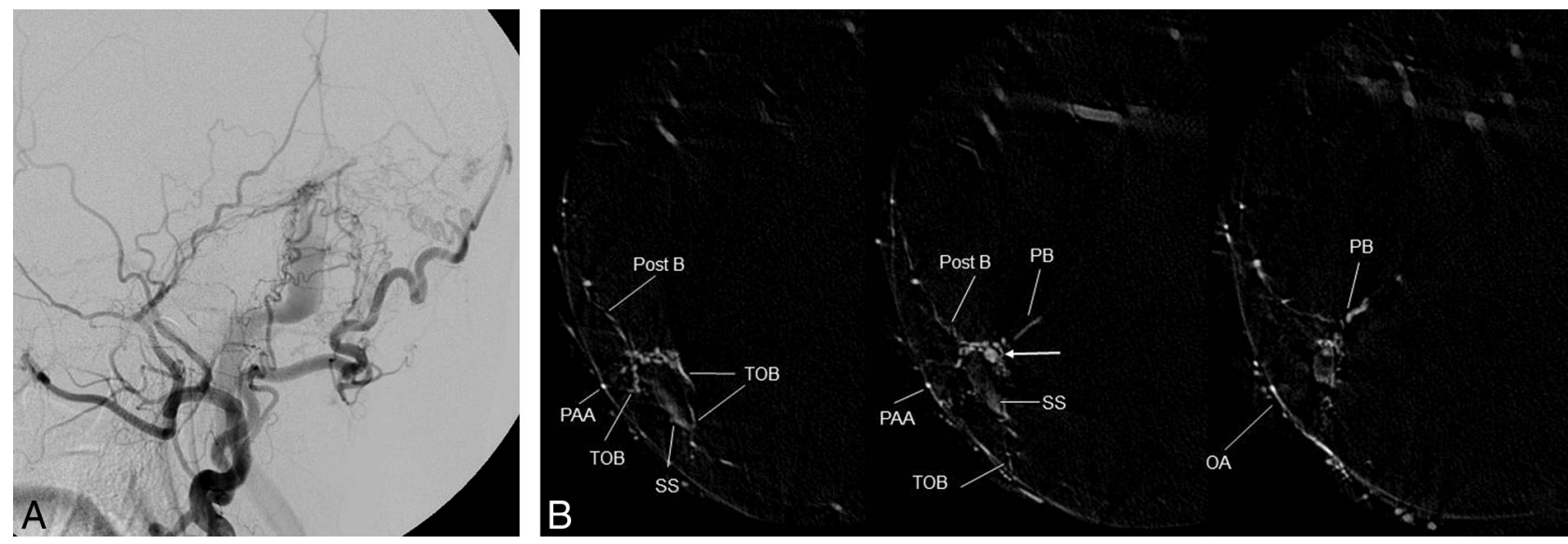

FIG 5. Right transverse sigmoid sinus DAVFs (type I) with a localized shunted venous pouch at the transverse-sigmoid junction. $A$, Right external carotid angiography shows DAVFs at the transverse-sigmoid junction. The DAVFs are fed by the right occipital artery, the posterior auricular artery, the posterior convexity branch, and the petrosal branch of the middle meningeal artery and drain antegradely into the sigmoid sinus. $B$, Axial reformatted images of DSA of the right external carotid artery show a shunted pouch (white arrow) that is located medial to the transverse-sigmoid junction. The shunted pouch is fed by the petrosal branch (PB) and the posterior convexity branch (Post B) of the middle meningeal artery, the posterior auricular artery (PAA), and the transosseous branches (TOB) of the occipital artery (OA). The AVFs disappeared following selective transvenous embolization (not shown).

packing and would be safer and more effective than simple sinus packing. Transarterial embolization with liquid embolic materials such as $n$-butyl 2-cyanoacrylate and Onyx (ev3, Irvine, California) is also a useful technique that has a high success rate in the treatment of intracranial DAVFs, according to several reports. ${ }^{9,10}$ However, transarterial embolization with liquid embolic materials via a dangerous feeder has a risk of complication because some of the feeding arteries of TSS-DAVFs, including the petrosal branch of the middle meningeal artery, the hypoglossal and jugular branches of the ascending pharyngeal artery, and the stylomastoid branch of the occipital and posterior auricular arteries, not only have anastomoses with the internal carotid artery, the vertebral artery, and the cerebellar arteries but also supply the cranial nerves. $^{11}$

There are close relationships between the locations of the shunted pouches and the feeding arteries in our cases. The shunted pouches located at the transverse-sigmoid junction were often supplied by the petrosal branch of the middle meningeal artery, and the shunted pouches located around the sigmoid sinus were fed by the jugular branch of the ascending pharyngeal artery. Selective transvenous embolization of these shunted pouches fed by dangerous feeders would be safer and more useful for the treatment of TSS-DAVFs. In our series, half of the cases were successfully treated by selective transvenous embolization alone. Transarterial embolization with $n$-BCA was required after selective transvenous embolization in the remaining 6 cases. In those 6 cases, coils placed in the shunted pouches by transvenous embolization were useful for preventing intrasinusal migration of $n$ BCA injected during transarterial embolization.

\section{CONCLUSIONS}

Most TSS-DAVFs are associated with shunted pouches and their proper feeding arteries. Knowledge of these angioarchitectures and a precise evaluation by angiography would be helpful for the treatment of TSS-DAVFs.

\section{REFERENCES}

1. Borden JA, Wu JK, Shucart WA. A proposed classification for spinal and cranial dural arteriovenous fistulous malformations and implications for treatment. J Neurosurg 1995;82:166-79

2. Cognard C, Gobin YP, Pierot L, et al. Cerebral dural arteriovenous fistulas: clinical and angiographic correlation with a revised classification of venous drainage. Radiology 1995;194:671-80

3. Mironov A. Selective transvenous embolization of dural fistulas without occlusion of the dural sinus. AJNR Am J Neuroradiol 1998; 19:389-91

4. Ohtakara K, Murao K, Kawaguchi K, et al. Selective transvenous liquid embolization of a type 1 dural arteriovenous fistula at the junction of the transverse and sigmoid sinuses: case report. $J \mathrm{Neu}$ rosurg 2000;92:1045-49

FIG 4. Left transvers sinus DAVFs (type lla $+b$ ) with left sigmoid sinus occlusion. Frontal $(A)$ and lateral $(B$ and $C$ ) views of the left external carotid angiography show DAVFs diffusely involving the left transverse sinus. The DAVFs are fed by the left occipital artery and the convexity branch of the middle meningeal artery and drain retrogradely into the contralateral transverse sinus, the straight sinus, and the left temporal vein (vein of Labbé). D, Frontal view of the right external carotid artery shows DAVFs at the sinus confluence fed by multiple branches of the occipital artery. E, Axial reformatted images of rotational angiography of the left external carotid artery show shunted pouches (white arrows) that are located inferodorsally to the distal transverse sinus. The shunted pouches were fed by numerous transosseous branches of the occipital artery (TOB). F, Axial reformatted images of rotational angiography of the right external carotid artery show another shunted pouch (white arrows) that is located inferodorsally to the sinus confluence. The shunted pouches were fed by multiple TOBs. G, Selective venography via a microcatheter navigated through the occluded sigmoid sinus shows a shunted venous pouch inferior to the transverse sinus. Each shunted pouch was selectively catheterized and embolized with coils. Right $(H)$ and left $(I)$ external carotid angiography after selective transvenous embolization shows the disappearance of the DAVFs. J, Left internal carotid angiography during the venous phase 10 days after embolization shows coils placed inferior to the transverse sinus and preserved and reconstructed venous flow in the transverse-sigmoid sinus. Note the normalized venous flow of the vein of Labbé antegradely into the transverse sinus. 

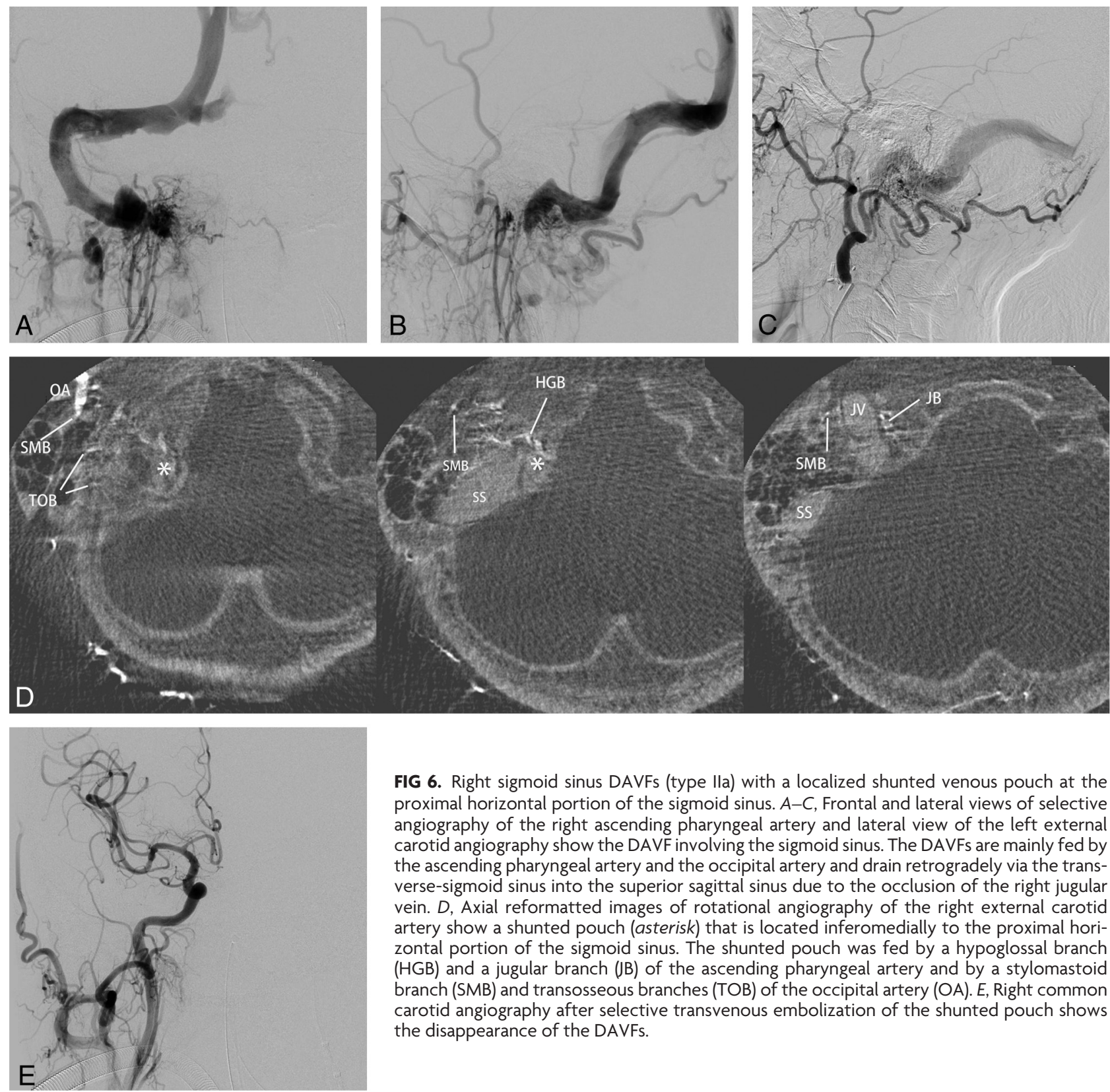

FIG 6. Right sigmoid sinus DAVFs (type Ila) with a localized shunted venous pouch at the proximal horizontal portion of the sigmoid sinus. $A-C$, Frontal and lateral views of selective angiography of the right ascending pharyngeal artery and lateral view of the left external carotid angiography show the DAVF involving the sigmoid sinus. The DAVFs are mainly fed by the ascending pharyngeal artery and the occipital artery and drain retrogradely via the transverse-sigmoid sinus into the superior sagittal sinus due to the occlusion of the right jugular vein. $D$, Axial reformatted images of rotational angiography of the right external carotid artery show a shunted pouch (asterisk) that is located inferomedially to the proximal horizontal portion of the sigmoid sinus. The shunted pouch was fed by a hypoglossal branch (HGB) and a jugular branch (JB) of the ascending pharyngeal artery and by a stylomastoid branch (SMB) and transosseous branches (TOB) of the occipital artery (OA). E, Right common carotid angiography after selective transvenous embolization of the shunted pouch shows the disappearance of the DAVFs.

5. Caragine LP, Halbach VV, Dowd CF, et al. Parallel venous channel as the recipient pouch in transverse/ sigmoid sinus dural fistulae. Neurosurgery 2003;53:1261-67, discussion 1266-67

6. Piske RL, Campos CMS, Chaves JBL, et al. Dural sinus compartment in dural arteriovenous shunts: a new angioarchitectural feature allowing superselective transvenous dural sinus occlusion treatment. AJNR Am J Neuroradiol 2005;26:1715-22

7. Hiu T, Kitagawa N, Morikawa M, et al. Efficacy of DynaCT digital angiography in the detection of the fistulous point of dural arteriovenous fistulas. AJNR Am J Neuroradiol 2009;30:487-91

8. Paget $\mathrm{DH}$. The cranial venous system in man in reference to devel- opment, adult configuration, and relation to the arteries. Am J Anat 1956;98:307-55

9. Kim DJ, Willinsky RA, Krings T, et al. Intracranial dural arteriovenous shunts: transarterial glue embolization-experience in 115 consecutive patients. Radiology 2011;258:554-61

10. van Rooij WJ, Sluzewski M. Curative embolization with Onyx of dural arteriovenous fistulas with cortical venous drainage. AJNR Am J Neuroradiol 2010;31:1516-20

11. Lv X, Jiang $\mathrm{C}$, Li Y, et al. The limitations and risks of transarterial Onyx injections in the treatment of grade I and II DAVFs. Eur J Radiol 2011;80:e385-88 